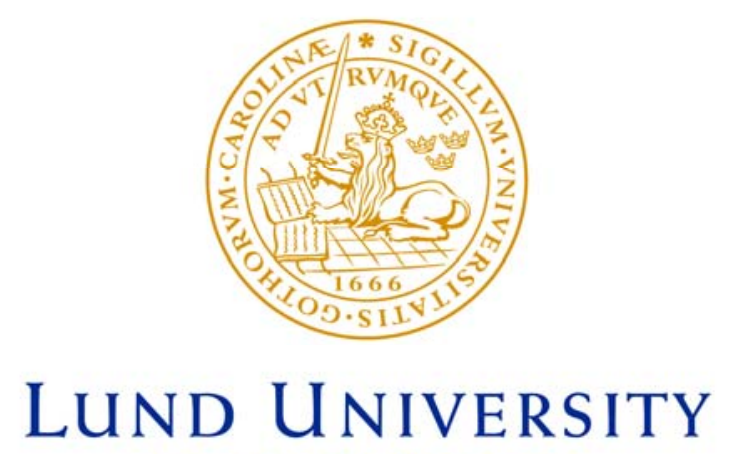

Faculty of Medicine

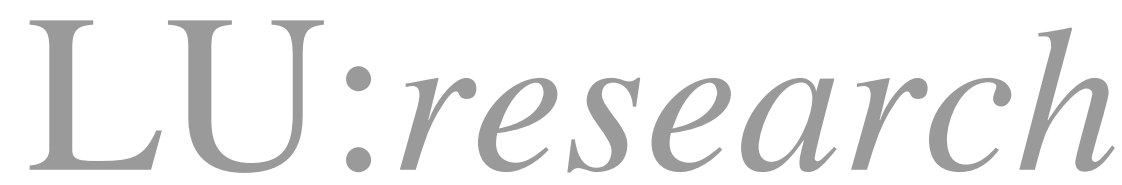

Institutional Repository of Lund University

This is an author produced version of a paper published in Osteoporosis International. This paper has been peerreviewed but does not include the final publisher proofcorrections or journal pagination.

Citation for the published paper:

Lenora, J and Ivaska, K and Obrant, K and Gerdhem, P. "Prediction of bone loss using biochemical markers of bone turnover"

Osteoporos Int, 2007, Vol:19, Issue:9, pp.1297-305.

http://dx.doi.org/10.1111/j.1365-2702.2006.01382.x

Access to the published version may require journal subscription.

Published with permission from: Springer 


\section{Prediction of bone loss using biochemical markers of bone turnover}

\section{Janaka Lenora $^{1}$, Kaisa K. Ivaska ${ }^{1}$, Karl J. Obrant ${ }^{1}$, Paul Gerdhem ${ }^{1,2}$.}

${ }^{1}$ Clinical and Molecular osteoporosis research unit, Department of Orthopaedics, Malmö University Hospital, Lund University, SE-205 02 Malmö, and ${ }^{2}$ Department of Orthopaedics, Karolinska University Hospital, Karolinska Institute, SE-141 86 Stockholm, Sweden

Correspondence: Janaka Lenora, Clinical and Molecular osteoporosis research unit, Department of Orthopaedics, Malmö University Hospital, Lund University, SE-205 02

Malmö, Sweden. Phone: +46 4033 7517. Fax: +46 40336933.

E-Mail: Robolge.Lenora@med.lu.se

\section{Word count}

Mini-Abstract: 48

Abstract: 200

Body of manuscript (excluding abstract): 3697

Number of tables: 4

Number of references: 38

Conflict of interests: None of the authors has a conflict of interest.

\section{Summary}

The association between baseline levels of eleven bone turnover markers and 5-year rate of bone density change was prospectively studied in a population-based sample of 601 , 75-yearold women. Several bone formation and resorption markers as well as urinary osteocalcin were modestly correlated to rate of bone density change. 


\section{Abstract}

Introduction: Prediction of bone loss by bone turnover markers (BTMs) has been investigated with conflicting results. There is limited information in the elderly.

Methods: Eleven bone turnover markers were analyzed in 75-year old women in the OPRA study ( $n=601)$ and compared to the 5-year change of areal bone mineral density (aBMD) in seven skeletal regions.

Results: Annual aBMD change varied between $+0.4 \%$ (spine) and $-2.0 \%$ (femoral neck). Significant associations $(\mathrm{p}<0.01)$ was found for four different serum osteocalcins (S-OCs) (standardized regression coefficient -0.20 to -0.22$)$, urinary deoxypyridinoline $(-0.19)$, serum TRACP5b (-0.19), serum CTX-I (-0.21), two of the three urinary osteocalcins (U-OCs) (0.16) and aBMD change of the leg region (derived from the total body measurement). After adjustment for baseline aBMD, associations were found for all S-OCs (-0.11 to -0.16$)$, two of the three U-OCs (-0.14 to -0.16) and aBMD change at the total hip, and for three of the four S-OCs (-0.14 to -0.15), S-TRACP5b (-0.11), two of the three U-OCs $(-0.14$ to -0.15$)$ and aBMD change at the femoral neck. There were no significant results concerning aBMD change at the spine.

Conclusion: This study indicates that BTM:s are correlated with aBMD loss in some skeletal regions in elderly women.

\section{Key words}

Bone density, bone turnover markers, bone loss, elderly. 


\section{Introduction}

Fractures can be predicted, at least on a population level, by measuring areal bone mineral density (aBMD) [1, 2]. There is also evidence that measuring bone metabolism by means of biochemical markers of bone turnover can predict fractures independently of bone mass [3-5]. There are some data indicating that the rate of bone loss itself is also predictive of fracture [6, 7].

The usefulness of bone turnover markers for predicting bone loss has been studied for the past two decades. Many of these studies were done in conjunction with tests of new pharmaceutical drugs $[8,9]$. Other studies involved mainly young women with a wide age range [10-15], unhealthy individuals [16], or limited number of subjects $[10,17,18]$, with only a short follow-up after sampling [10, 12]. Knowledge based on studies of large populations, with long follow up periods is, therefore, limited.

The aim of this study was to evaluate the ability of eleven bone turnover markers, including recently developed assays for the analysis of urine osteocalcin and tartrate-resistant acid phosphatase 5b, to predict 5-year rate of aBMD loss in a population-based sample of elderly women.

\section{Individuals and methods}

\section{Individuals}

The Malmö Osteoporosis Prospective Risk Assessment (OPRA) study is an ongoing longitudinal study of elderly women in Malmö, Sweden. At inclusion all women were 75 years old, and follow-ups have taken place at the ages of 76, 78 and 80. Bone density was measured at the 75 and 80-year follow-up. For the baseline investigation, a letter of invitation 
was sent out one week after her $75^{\text {th }}$ birthday to 1604 women, all randomly selected from the city files of Malmö, Sweden. The baseline investigations took place between 1995 and 1999, and 1044 women chose to attend (65\%). Of the 560 women who did not participate, 13 died shortly after the invitation, 139 could not come because of illness, 376 were not interested or could not attend for reasons other than illness and 32 women were not reached despite repeated letters and phone calls [19].

Out of the 1044 women participating in the study, areal bone mineral density (aBMD) measurements could not be performed in 49 women; 40 women were unable to visit the research department and 9 women could not be measured due to technical problems in the bone mass measurement (high body weight, disability, not allowing the supine positioning for the time required for measurement or prior surgery interfering with the measurements). Baseline aBMD was performed in 995 individuals. Of these, 119 had been taking bone active drugs, oestrogen or bisphosphonates, at anytime during the 5-year follow up period. Thus, 876 women remained for the study at baseline.

Of the 876 women participating at baseline 601 women returned for follow-up at a mean of 5.0 years later (range $4.9-5.4$ years). The percentage completing the investigation was thus $69 \%$.

All parts of the study were approved by the ethics committee of Lund University.

\section{Bone density}

The areal bone mineral density (aBMD) of the total body, femoral neck, total hip and lumbar spine ( $\mathrm{L}_{2}$ to $\mathrm{L}_{4}$ ), was measured by dual-energy x-ray absorptiometry (DXA) (Lunar DPX-L® 
Madison, USA). Also three specific regions of interest (RoI) derived from the total body measurement were assessed; the arms, the legs and the partial body. The partial body measurement was the total body measurement in which the head regional measurement had been subtracted. Scan analysis at baseline was made with software versions 1.33 and 1.35 and at five years with software version 4.7b, with the exception of hip scans, which were all analyzed with software version $4.7 \mathrm{~b}$.

The precision of the DXA equipment as assessed by duplicate measurements after repositioning [20] in 15, 80-year old volunteers of the OPRA study was $0.5 \%$ for the total body, $1.5 \%$ for the partial body, $0.90 \%$ for the legs, $1.8 \%$ for the arms, $3.6 \%$ for the total hip, $3.9 \%$ for the femoral neck and $1.2 \%$ for the spine. The stability of the DPX-L equipment was checked every morning using a phantom provided by the manufacturer. There was no drift in phantom measurement results during the study period.

\section{Serum and urine samples}

Serum and urine samples were collected for the analysis of markers of bone turnover at baseline. Blood samples were collected non-fasting between 08.00-13.00 and centrifuged within 2 hours after phlebotomy. First morning void urine samples were collected. Serum and urine samples were stored at $-80^{\circ} \mathrm{C}$. All the analyses for each bone metabolic marker were done at the same time in order to minimize inter-assay variability. Since the women were recruited during the years 1995 to 1999, sample storage times were up to 3.6 years apart.

\section{Markers of bone formation}

Inter- and intra-assay coefficients of variation (CV) for each assay have been determined previously [3]. Bone-specific alkaline phosphatase (S-Bone ALP) was determined by using 
Metra BAP immunoassay (Quidel Corporation), with an intra- and inter- assay CV of 3.6\% and 4.4\%, respectively. Serum intact and N-mid Osteocalcin (S-Total OC(N-Mid®)) were determined by using the Elecsys N-MID Osteocalcin immunoassay (S-Total OC; N-MID®; Roche Diagnostics), with intra- and inter-assay CV of $2.3 \%$ and 2.4\%, respectively. Serum intact osteocalcin (S-OC [1-49]), serum total osteocalcin (S-Total OC) and serum total carboxylated osteocalcin (S-cOC) were determined by previously described, in-house protocols with intra- and inter- assay CV of less than $5 \%$ and $8 \%$, respectively, for all the assays [21]. Briefly, protocols are two-site assays based on two monoclonal antibodies (Mabs) in combinations 3G8/2H9 (S-OC[1-49]), 2H9/6F9 (S-TotalOC) and 6F9/3H8 (S-cOC). Mab $3 \mathrm{G} 8$ is specific for intact OC, Mab $6 \mathrm{H} 9$ binds to fragment $\mathrm{Gly}^{7}-\mathrm{Arg}^{19}$, Mab 2H9 recognizes fragment $\mathrm{Arg}^{20}-\mathrm{Arg}^{43}$ and Mab $3 \mathrm{H} 8$ binds to the same fragment $\mathrm{Arg}^{20}-\mathrm{Arg}^{43}$ but prefers OC containg gammacarboxyglutamic acid (Gla), with only 9\% cross-reactivity to non-Glacontaining OC [22].

\section{Markers of bone resorption}

Serum C-terminal cross-linking telopeptides of type I collagen (S-CTX-I) was determined by Elecsys $\beta$-Cross Laps ${ }^{\circledR}$ immunoassay (Roche diagnostics) with intra- and inter- assay CV of $5.9 \%$ and $5.8 \%$, respectively. Serum tartrate-resistant acid phosphatase 5b (S-TRACP5b) was assessed by a solid phase, immunofixed, enzyme activity assay as described earlier [23]with an intra- and inter- assay CV of $1.8 \%$ and $2.2 \%$, respectively.

Urinary deoxypyridinoline (U-DPD) was measured by the Metra DPD immunoassay (Quidel Corporation) with an intra- and inter- assay CV less than $12 \%$ and $10 \%$, respectively. 


\section{Urinary osteocalcin}

Urinary osteocalcin (U-OC) consists of fragments less than thirty residues in length from the middle region of the molecule [24]. Three assays for the detection of various molecular forms of U-OC were analyzed as described previously [25]. Assays were based on the same Mabs as the assays for serum OC (see details above). Briefly, two-site assay U-MidOC consists of Mabs 6F9 and $3 \mathrm{H} 8$ and recognizes the most abundant midmolecule fragments of U-OC (spanning residues 7-31, 7-29, 6-29, 9-31, 7-32 and 7-33). Two-site assay U-LongOC (2H9/6F9) detects only the longest U-OC fragments (7-32, 7-33) with low affinity. Competitive assay U-TotalOC (3H8) measures (in addition to the same midmolecule fragments) also more truncated U-OC fragments, starting from residue Asp ${ }^{14}$. The intra- and inter- assay CVs were 1.7\% and < 12\%(U-MidOC), $4.3 \%$ and < 14\% (U-LongOC), and 14\% and $<27 \%$ (U-TotalOC), respectively [25].

\section{Urinary creatinine}

Urinary creatinine was measured by the kinetic Jaffe reaction with a Beckman synchron LX20-4, with CVs of $3 \%$ or less. All the measurements of urinary bone markers were corrected for urinary creatinine and expressed as ratios.

\section{Other data}

The history of bone active medication was assessed by use of a questionnaire and crosschecked by one of the investigators at baseline, at the 1-year (age 76), 3-year (age 78) and the 5-year (age 80) follow-up. Fractures during the follow up period were identified by questionnaire and by hospital reports, as reported previously [3]. 


\section{Statistical analysis}

Statistica for Windows (version 7.1, Stat Soft Inc) software was used for the statistical analysis. The DXA variables were found to have an approximately normal distribution (Shapiro-Wilks test $>0.97$ ). Logarithmic transformation of the bone turnover markers was made since it resulted in a more normal distribution (Shapiro-Wilks test $>0.91$ ).

In the regression calculations, the annual rates of aBMD loss of the different skeletal sites were set as dependent variables. The baseline levels of the eleven markers of bone turnover were compared using a standardized regression coefficient (Beta std $_{\text {) }}$ both before and after adjustment for the baseline aBMD or total body BMC. The standardization makes it possible to compare the results between different markers and also utilizes all the available data. In a separate calculation adjustment for sample storage times was made.

In addition to the regression calculations, quartile comparisons were also made. The women were divided into quartiles according to bone marker level and yearly aBMD change. Odds ratios and t-tests were used to compare groups of women.

When there is dependency between bone turnover markers and between BMD measurements of different skeletal sites, it is not possible to calculate the exact adjustment for multiple testing. Therefore the level of significance was regarded throughout the entire investigation as statistically significant finding when $\mathrm{p}<0.01$ to avoid mass significances. 


\section{Results}

There was a decrease of bone density in all measured skeletal regions except the lumbar spine, during the five-year follow-up period. The yearly aBMD change varied between -2.0 \% (femoral neck) and $+0.4 \%$ (lumbar spine) (Table 1). The mean (SD) baseline levels of bone turnover markers are also given in table 1. During the follow-up period 104 out of the 601 women sustained at least one incident fracture.

The individuals who completed the study and the individuals who attended only for the baseline investigation were compared. There was no significant difference in age, height and weight. The individuals who attended only baseline had lower aBMD than the ones who completed the whole study, at the leg region ( 0.99 vs. $1.01, \mathrm{p}=0.03)$, at the total hip ( 0.83 vs. 0.87, $\mathrm{p}<0.001)$, and the femoral neck (0.75 vs. 0.78, $\mathrm{p}=0.003)$. The individuals who attended only baseline had higher levels of U-DPD/crea (8.9 vs. $8.2 \mathrm{p}=0.01$ ) and U-LongOC/crea ( 0.084 vs. $0.052 \mathrm{p}=0.02$ ) than the individuals who completed the study. The regional aBMD results of the other sites and other bone turnover markers were not different between the two groups.

\section{Standardized regression calculations}

The baseline levels of all serum osteocalcins were significantly associated with aBMD loss of the leg region, with the unadjusted Beta ${ }_{\text {std }}$ varying between -0.20 and -0.22 . S-OC [1-49], STotal OC and S-cOC were significantly associated with partial body aBMD loss with a Beta std of -0.12 for all these formation markers. S-Bone ALP was not significantly associated to aBMD change at any site (Table 2). 
The baseline levels of U-DPD/crea, S-TRACP5b and S-CTX-I were significantly associated

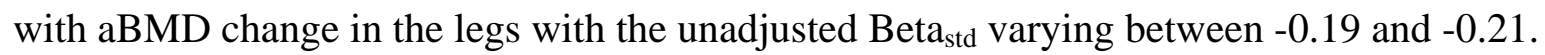
U-DPD/crea and S-CTX-I were also significantly associated with aBMD change in the partial body and U-DPD/crea with aBMD change in the total body. There was no association between the resorption markers and aBMD change at any other skeletal site (Table 2).

The Beta ${ }_{\text {std }}$ between the urinary osteocalcins U-LongOC/crea and U-MidOC/crea and aBMD change in the legs were for both markers -0.16. U-Mid OC was also associated to aBMD change in the partial body measurement (Table 2).

When the regression calculations were performed after adjusting for baseline levels of aBMD, statistically significant associations between S-OC [1-49], S-Total OC, S-cOC, U-DPD/crea, S-CTX-I and S-TRACP5b and the rate of aBMD change in the arms, the femoral neck or the total hip was found (Table 3). When instead adjustment for total body BMC was made, the regression results for aBMD changes at the leg region, the total body or the partial body was not substantially affected. The regression results for aBMD loss at the total hip and the femoral neck became non-significant after adjustment for total body BMC (data not shown). Adjustment for incident fractures did not substantially change the associations between baseline marker levels and rate of bone loss (data not shown).

Adjustment for storage time increased the Beta $_{\text {std }}$ to significant levels for the following associations: U-TotalOC/crea and bone density change of the legs $(-0.14, p=0.002)$ and STRACP5b and partial body bone density change $(-0.13, \mathrm{p}=0.002)$. The associations between S-Total OC, S-OC [1-49], S-cOC and U-MidOC/crea and bone density change in the partial 
body measurement were no longer significant after adjustment for sample storage times (data not shown).

\section{Quartile comparisons}

Women within the highest quartile of any of the S-OCs had higher bone loss in the leg region when compared to women in the other three quartiles. Women within the highest quartile of S-CTX-I and U-MidOC/crea had significantly higher aBMD loss in the leg region when compared to the other women. Significant differences concerning bone loss was also seen for some of the S-OCs in other aBMD regions. Significant differences concerning bone loss was seen for U-DPD/crea in 4 of the 7 aBMD regions (Table 4).

The risk for the group of women with a bone turnover marker level in the highest quartile to also be in the highest quartile of bone loss during the five-year follow-up, compared to all the other women, was analyzed with odds ratios (OR). The women in the highest quartile of STotal OC (N-Mid $\left.{ }^{\circledR}\right)$ had an OR of 1.79 (99\% confidence limit 1.01-3.16) for being in the highest quartile of bone loss at the leg region, and an OR of 1.78 (1.01-3.09) to be in the highest quartile of bone loss in the femoral neck. Corresponding results for S-Total OC and rate of bone loss in the leg region was 1.79 (1.02-3.14). Results for S-cOC were 1.97 (1.123.44) for the leg region, 1.88 (1.07-3.28) for the total body, and 2.19 (1.25-3.82) for the partial body. The OR for U-DPD/crea for bone loss in the total body was 1.79 (1.02-3.15).

Corresponding OR results for S-CTX-I were 2.16 (1.23-3.81) for the leg region and 1.81(1.02-3.20) for the partial body. The results for U-Mid OC/crea were 1.97 (1.28-3.03) for the leg region and 1.94 (1.01-3.41) for the partial body. The results for other BTMs and other regions were not significant (data not shown). 


\section{Discussion}

In this study of elderly women, all markers correlated to areal bone mineral density change in a large weight bearing region (the legs), with the exceptions of S-Bone ALP and U-Total OC/crea. The S-OCs, S-TRACP5b and two of the U-OCs were to some extent correlated to aBMD change at the hip after correction for baseline aBMD. Standardized regression was used to facilitate comparison between different markers. However, there was no clear superiority of any specific marker, or group of markers, in the association to bone loss.

To the best of our knowledge, this is the largest study on bone turnover markers and bone loss in elderly women. The women have been prospectively followed for a long period (5 years) and bone turnover has been assessed by several bone formation and resorption markers. The associations between TRACP5b, urinary osteocalcins, and bone loss have not been reported earlier.

Tartrate resistant acid phosphatase exists in two isoforms, 5a and 5b. TRACP5b is secreted by osteoclasts. TRACP5b has recently been described as a specific and sensitive serum marker of bone resorption [26, 27], and to correlate with other markers of bone turnover and bone mineral density [28]. We have earlier reported that S-TRACP5b had a fracture predictive ability in the OPRA-study [3]. In the present study, S-TRACP5b was correlated to aBMD change in the legs, and after adjustment for baseline aBMD, to aBMD change at the femoral neck.

If the urinary osteocalcins are markers of resorption or formation have not yet been fully elucidated. Previous studies indicate that they may represent bone resorption [25, 29, 30]. 
U-LongOC/crea and U-MidOC/crea were to some extent associated to aBMD change, while U-TotalOC/crea had no association to aBMD change at any region.

Deoxy-pyridinoline is an important cross-link in collagen and increases its tensile strength [31]. It is released during degradation of bone. U-DPD had correlations to more skeletal regions (legs, partial body, total body, and after adjustment for baseline aBMD, arms) than the other resorption markers. U-DPD has been studied extensively earlier concerning the ability to predict bone loss with positive findings concerning the ability to predict hip bone loss [13, 17, 32, 33] and spine bone loss [10, 33].

CTX-I is generated in the degradation of bone collagen and can be measured in urine or serum. To the best of our knowledge, S-CTX-I has not been investigated for the prediction of bone loss earlier. In the present study, S-CTX-I correlated to aBMD change in the legs, partial body, and after adjustment for baseline aBMD, arms. The prediction of bone loss with CTX-I analyzed in urine (U-CTX-I) has been studied in some reports. Correlations between U-CTX-I levels and hip or spine bone loss have been reported by some authors [8, 13, 33, 34].

Bone formation was assessed with serum osteocalcin and serum bone specific alkaline phosphatase. Osteocalcin is quickly degraded in the circulation and several different forms can be found in serum [31]. In the present study we have used four different serum osteocalcin assays, but without finding any substantial differences concerning the ability to predict bone loss. After correction for baseline aBMD, all the S-OCs correlated to aBMD loss in the total hip. S-OC has been reported to be associated with hip bone loss in elderly women (mean ages 73 and 71, respectively) [13, 17] and in perimenopausal women [12, 33], and in the spine in reports including heterogeneous groups of women [8, 32]. 
Bone alkaline phosphatase is important for osteoid formation and mineralization [31]. S-Bone ALP did not correlate to bone density change in any skeletal region in the present study. Earlier reports have been inconsistent. S-Bone ALP was not associated to hip bone loss in a study by Chapurlat et al [12]. However, in a subgroup analysis of perimenopausal women with increased levels of follicle stimulating hormone there was an association to hip bone loss [12]. Dresner-Pollak et al reported an association between S-Bone ALP and hip bone loss in elderly women (mean age 71) [17]. Baseline levels of S-Bone ALP has been reported to be correlated to spine bone loss in various groups of women [10, 32, 33, 35].

For most of the bone turnover markers there was a correlation between baseline level and aBMD change in the legs, a large weight bearing region derived from the total body scan, representing a large part of bone metabolism. The S-OCs, two of the three urinary OCs, and to some extent S-TRACP5b, were significantly associated to bone density loss in the hip (femoral neck or total hip) after correction for baseline aBMD. When the total hip is used instead of the femoral neck, the error caused by the presence of osteophytes in the femoral neck is to some extent masked by the larger volume of bone included. Others have found better correlations between BTMs and total hip bone density change than to femoral neck bone density change $[13,17]$. However, in this study, there was no substantial difference between the ability of BTMs to predict bone density changes at the total hip or femoral neck region.

The reason for the lack of an association for aBMD in the spine may be due to several reasons. Age-related changes such as the appearance of osteophytes, which are not uncommon at this age, affect the BMD. At higher ages spinal vertebral compression fractures, 
osteophytes and aortic calcification may mask aBMD loss. For these reasons it is understandable that the lumbar spine BMD was increased during the 5-year follow-up period.

In general, weak associations between bone turnover markers and aBMD change were seen in this large study of elderly women, and can explain the lack of significant associations in some previous studies [12, 17, 18, 36]. It seems that correlations between bone turnover markers, both formation and resorption, are stronger when women in the perimenopausal, or early postmenopausal, period are studied, as reported by Iki and co-workers [33]. Study cohorts with older subjects report lower correlations between marker levels and bone density change $[10,13,33]$ than studies with younger subjects $[8,10,12,33]$.

We believe the particular strengths of this study are its prospective design, with a 5-year follow-up, the large cohort of randomly selected women of the same age, BMD assessed at multiple skeletal sites, and a large number of markers assessed. There are also some limitations. Since all women were of the same age and ethnic background, caution must be exercised when the results are transferred to other than 75-year old Caucasian women. We find no reason, however, to believe that the results should not be applicable to other women well above menopausal age.

Previous studies have shown that markers of bone resorption are more affected by circadian variation and feeding than formation markers [37]. When this study was designed this knowledge was not available. Serum sampling have been done both fasting and non-fasting in earlier reports, with no clear association between fasting status and study results $[8,10,18$, 38]. To minimize circadian variation it is important to minimize the sampling period of the day, which was also done in this study. The correlation between sampling time of the day and 
the bone turnover marker level was less than 0.07 for all bone turnover markers, as reported earlier in the OPRA- study [3]. The samples were analyzed at the same time in order to minimize inter-assay variability. Since it took several years to recruit the participants the storage time for the samples varied. However, when storage times were taken into account in the calculations there were only minor changes in the results. The coefficient of variation for bone turnover markers could influence the bone loss predictive ability. Increasing the number of occasions for sampling could possibly increase precision and might increase the ability to predict bone density change.

In summary, from this large cohort of randomly selected elderly women and after exclusion of women on bone-active drugs, we conclude that markers of bone metabolism are associated to aBMD loss in some skeletal sites. However, there was no clear prediction of bone loss at the clinically important sites, the hip and the lumbar spine, limiting the utility of bone turnover markers as predictors of bone loss.

\section{Acknowledgements}

Financial support was received from the Swedish Medical Research Council, Malmö University Hospital Funds, and the Greta and Johan Kock Foundation. 


\section{References}

1. Johnell O, Kanis JA, Oden A, Johansson H, De Laet C, Delmas P, Eisman JA, Fujiwara S, Kroger H, Mellstrom D, Meunier PJ, Melton LJ, 3rd, O'Neill T, Pols H, Reeve J, Silman A, Tenenhouse A (2005) Predictive value of BMD for hip and other fractures. J Bone Miner Res 20:1185-1194

2. Stewart A, Kumar V, Reid DM (2006) Long-term fracture prediction by DXA and QUS: a 10-year prospective study. J Bone Miner Res 21:413-418

3. Gerdhem P, Ivaska KK, Alatalo SL, Halleen JM, Hellman J, Isaksson A, Pettersson K, Vaananen HK, Akesson K, Obrant KJ (2004) Biochemical markers of bone metabolism and prediction of fracture in elderly women. J Bone Miner Res 19:386393

4. Garnero P, Hausherr E, Chapuy MC, Marcelli C, Grandjean H, Muller C, Cormier C, Breart G, Meunier PJ, Delmas PD (1996) Markers of bone resorption predict hip fracture in elderly women: the EPIDOS Prospective Study. J Bone Miner Res 11:1531-1538

5. Garnero P, Sornay-Rendu E, Claustrat B, Delmas PD (2000) Biochemical markers of bone turnover, endogenous hormones and the risk of fractures in postmenopausal women: the OFELY study. J Bone Miner Res 15:1526-1536

6. Sornay-Rendu E, Munoz F, Duboeuf F, Delmas PD (2005) Rate of forearm bone loss is associated with an increased risk of fracture independently of bone mass in postmenopausal women: the OFELY study. J Bone Miner Res 20:1929-1935

7. Nguyen TV, Center JR, Eisman JA (2005) Femoral neck bone loss predicts fracture risk independent of baseline BMD. J Bone Miner Res 20:1195-1201

8. Marcus R, Holloway L, Wells B, Greendale G, James MK, Wasilauskas C, Kelaghan J (1999) The relationship of biochemical markers of bone turnover to bone density changes in postmenopausal women: results from the Postmenopausal Estrogen/Progestin Interventions (PEPI) trial. J Bone Miner Res 14:1583-1595

9. Sarkar S, Reginster JY, Crans GG, Diez-Perez A, Pinette KV, Delmas PD (2004) Relationship between changes in biochemical markers of bone turnover and BMD to predict vertebral fracture risk. J Bone Miner Res 19:394-401

10. Rogers A, Hannon RA, Eastell R (2000) Biochemical markers as predictors of rates of bone loss after menopause. J Bone Miner Res 15:1398-1404

11. Lofman O, Magnusson P, Toss G, Larsson L (2005) Common biochemical markers of bone turnover predict future bone loss: a 5-year follow-up study. Clin Chim Acta 356:67-75

12. Chapurlat RD, Gamero P, Sornay-Rendu E, Arlot ME, Claustrat B, Delmas PD (2000) Longitudinal study of bone loss in pre- and perimenopausal women: evidence for bone loss in perimenopausal women. Osteoporos Int 11:493-498

13. Bauer DC, Sklarin PM, Stone KL, Black DM, Nevitt MC, Ensrud KE, Arnaud CD, Genant HK, Garnero P, Delmas PD, Lawaetz H, Cummings SR (1999) Biochemical markers of bone turnover and prediction of hip bone loss in older women: the study of osteoporotic fractures. J Bone Miner Res 14:1404-1410

14. Garnero P, Sornay-Rendu E, Duboeuf F, Delmas PD (1999) Markers of bone turnover predict postmenopausal forearm bone loss over 4 years: the OFELY study. J Bone Miner Res 14:1614-1621

15. Melton LJ, 3rd, Atkinson EJ, O'Connor MK, O'Fallon WM, Riggs BL (2000) Determinants of bone loss from the femoral neck in women of different ages. $J$ Bone Miner Res 15:24-31 
16. Stepan JJ, Havrdova E, Tyblova M, Horakova D, Ticha V, Novakova I, Zikan V (2004) Markers of bone remodeling predict rate of bone loss in multiple sclerosis patients treated with low dose glucocorticoids. Clin Chim Acta 348:147-154

17. Dresner-Pollak R, Parker RA, Poku M, Thompson J, Seibel MJ, Greenspan SL (1996) Biochemical markers of bone turnover reflect femoral bone loss in elderly women. Calcif Tissue Int 59:328-333

18. Dennison E, Eastell R, Fall CH, Kellingray S, Wood PJ, Cooper C (1999) Determinants of bone loss in elderly men and women: a prospective population-based study. Osteoporos Int 10:384-391

19. Obrant KJ, Ivaska KK, Gerdhem P, Alatalo SL, Pettersson K, Vaananen HK (2005) Biochemical markers of bone turnover are influenced by recently sustained fracture. Bone 36:786-792

20. Gluer CC, Blake G, Lu Y, Blunt BA, Jergas M, Genant HK (1995) Accurate assessment of precision errors: how to measure the reproducibility of bone densitometry techniques. Osteoporos Int 5:262-270

21. Kakonen SM, Hellman J, Karp M, Laaksonen P, Obrant KJ, Vaananen HK, Lovgren T, Pettersson K (2000) Development and evaluation of three immunofluorometric assays that measure different forms of osteocalcin in serum. Clin Chem 46:332-337

22. Hellman J, Kakonen SM, Matikainen MT, Karp M, Lovgren T, Vaananen HK, Pettersson K (1996) Epitope mapping of nine monoclonal antibodies against osteocalcin: combinations into two-site assays affect both assay specificity and sample stability. J Bone Miner Res 11:1165-1175

23. Halleen JM, Alatalo SL, Suominen H, Cheng S, Janckila AJ, Vaananen HK (2000) Tartrate-resistant acid phosphatase 5b: a novel serum marker of bone resorption. $\mathrm{J}$ Bone Miner Res 15:1337-1345

24. Ivaska KK, Hellman J, Likojarvi J, Kakonen SM, Gerdhem P, Akesson K, Obrant KJ, Pettersson K, Vaananen HK (2003) Identification of novel proteolytic forms of osteocalcin in human urine. Biochem Biophys Res Commun 306:973-980

25. Ivaska KK, Kakonen SM, Gerdhem P, Obrant KJ, Pettersson K, Vaananen HK (2005) Urinary osteocalcin as a marker of bone metabolism. Clin Chem 51:618-628

26. Halleen JM, Alatalo SL, Janckila AJ, Woitge HW, Seibel MJ, Vaananen HK (2001) Serum tartrate-resistant acid phosphatase $5 \mathrm{~b}$ is a specific and sensitive marker of bone resorption. Clin Chem 47:597-600

27. Janckila AJ, Takahashi K, Sun SZ, Yam LT (2001) Tartrate-resistant acid phosphatase isoform 5 b as serum marker for osteoclastic activity. Clin Chem 47:74-80

28. Halleen JM, Ylipahkala H, Alatalo SL, Janckila AJ, Heikkinen JE, Suominen H, Cheng S, Vaananen HK (2002) Serum tartrate-resistant acid phosphatase 5b, but not $5 \mathrm{a}$, correlates with other markers of bone turnover and bone mineral density. Calcif Tissue Int 71:20-25

29. Akesson K, Kakonen SM, Josefsson PO, Karlsson MK, Obrant KJ, Pettersson K (2005) Fracture-induced changes in bone turnover: a potential confounder in the use of biochemical markers in osteoporosis. J Bone Miner Metab 23:30-35

30. Srivastava AK, Mohan S, Singer FR, Baylink DJ (2002) A urine midmolecule osteocalcin assay shows higher discriminatory power than a serum midmolecule osteocalcin assay during short-term alendronate treatment of osteoporotic patients. Bone 31:62-69

31. Delmas PD, Eastell R, Garnero P, Seibel MJ, Stepan J (2000) The use of biochemical markers of bone turnover in osteoporosis. Committee of Scientific Advisors of the International Osteoporosis Foundation. Osteoporos Int 11 Suppl 6:S2-17 
32. Cosman F, Nieves J, Wilkinson C, Schnering D, Shen V, Lindsay R (1996) Bone density change and biochemical indices of skeletal turnover. Calcif Tissue Int 58:236243

33. Iki M, Morita A, Ikeda Y, Sato Y, Akiba T, Matsumoto T, Nishino H, Kagamimori S, Kagawa Y, Yoneshima H (2006) Biochemical markers of bone turnover predict bone loss in perimenopausal women but not in postmenopausal women-the Japanese Population-based Osteoporosis (JPOS) Cohort Study. Osteoporos Int 17:1086-1095

34. Bruyere O, Collette J, Delmas P, Rouillon A, Roux C, Seidel L, Richy F, Reginster JY (2003) Interest of biochemical markers of bone turnover for long-term prediction of new vertebral fracture in postmenopausal osteoporotic women. Maturitas 44:259-265

35. Iki M, Kajita E, Dohi Y, Nishino H, Kusaka Y, Tsuchida C, Yamamoto K, Ishii Y (1996) Age, menopause, bone turnover markers and lumbar bone loss in healthy Japanese women. Maturitas 25:59-67

36. Keen RW, Nguyen T, Sobnack R, Perry LA, Thompson PW, Spector TD (1996) Can biochemical markers predict bone loss at the hip and spine?: a 4-year prospective study of 141 early postmenopausal women. Osteoporos Int 6:399-406

37. Clowes JA, Hannon RA, Yap TS, Hoyle NR, Blumsohn A, Eastell R (2002) Effect of feeding on bone turnover markers and its impact on biological variability of measurements. Bone 30:886-890

38. Chapurlat RD, Garnero P, Breart G, Meunier PJ, Delmas PD (2000) Serum type I collagen breakdown product (serum CTX) predicts hip fracture risk in elderly women: the EPIDOS study. Bone 27:283-286 
Table 1. The means (SD) of aBMD and bone turnover markers at baseline. Only women assessed at baseline and at follow-up are included in this table. All the 5-year BMD changes are statistically significant $(\mathrm{p}<0.001)$. BMD after 5 years are also given.

\begin{tabular}{|c|c|c|c|c|}
\hline & $\mathrm{n}$ & $\begin{array}{c}\text { Baseline (at } 75 \\
\text { years) }\end{array}$ & $\begin{array}{c}5 \text { year (at } 80 \\
\text { years) }\end{array}$ & $\begin{array}{l}\text { Per cent (SD) } \\
\text { change /year }\end{array}$ \\
\hline \multicolumn{5}{|l|}{ BMD } \\
\hline Arms $\left(\mathrm{g} / \mathrm{cm}^{2}\right)$ & 549 & $0.73(0.09)$ & $0.70(0.08)$ & $-0.88(1.03)$ \\
\hline Legs $\left(\mathrm{g} / \mathrm{cm}^{2}\right)$ & 549 & $1.01(0.12)$ & $0.98(0.12)$ & $-0.57(0.74)$ \\
\hline Total body $\left(\mathrm{g} / \mathrm{cm}^{2}\right)$ & 549 & $1.01(0.09)$ & $1.00(0.10)$ & $-0.32(0.64)$ \\
\hline Partial body $\left(\mathrm{g} / \mathrm{cm}^{2}\right)$ & 549 & $0.89(0.09)$ & $0.87(0.10)$ & $-0.45(0.72)$ \\
\hline Femoral neck (g/cm²) & 573 & $0.78(0.13)$ & $0.72(0.13)$ & $-1.95(2.66)$ \\
\hline Total hip $\left(\mathrm{g} / \mathrm{cm}^{2}\right)$ & 556 & $0.87(0.15)$ & $0.81(0.14)$ & $-1.31(1.80)$ \\
\hline Lumbar spine $\left(\mathrm{g} / \mathrm{cm}^{2}\right)$ & 587 & $1.00(0.19)$ & $1.02(0.20)$ & $+0.37(1.46)$ \\
\hline \multicolumn{5}{|l|}{ Bone formation markers } \\
\hline S-Bone ALP (U/L) & 579 & $23.1(8.8)$ & - & - \\
\hline S-Total OC $\left(\mathrm{N}-\mathrm{Mid}{ }^{\circledR}\right)(\mu \mathrm{g} / \mathrm{L})$ & 579 & $29.5(12.0)$ & - & - \\
\hline S-OC [1-49] $(\mu \mathrm{g} / \mathrm{L})$ & 593 & $5.3(2.5)$ & - & - \\
\hline S-Total OC $(\mu \mathrm{g} / \mathrm{L})$ & 593 & $8.8(3.6)$ & - & - \\
\hline S-cOC $(\mu \mathrm{g} / \mathrm{L})$ & 593 & $7.6(3.0)$ & - & - \\
\hline \multicolumn{5}{|l|}{ Bone resorption Markers } \\
\hline S-TRACP5b (U/L) & 592 & $3.4(1.1)$ & - & - \\
\hline U-DPD/crea (nmol/mmol) & 590 & $8.2(3.5)$ & - & - \\
\hline S-CTX-I (ng/L) & 579 & $312(186)$ & - & - \\
\hline \multicolumn{5}{|l|}{ Urinary Osteocalcins } \\
\hline U-Total OC/Crea $(\mu \mathrm{g} / \mathrm{mmol})$ & 591 & $27.6(23.7)$ & - & - \\
\hline U-LongOC/crea $(\mu \mathrm{g} / \mathrm{mmol})$ & 591 & $0.05(0.05)$ & - & - \\
\hline U-MidOC/crea ( $\mu \mathrm{g} / \mathrm{mmol})$ & 591 & $1.3(0.8)$ & - & - \\
\hline
\end{tabular}


Table 2. Standardized regression coefficients (Beta $\mathrm{std}_{\mathrm{d}}$ ) between baseline levels of 11 bone markers and rate of bone loss at the different regions of interest, unadjusted for the baseline areal bone mineral density (aBMD).

Significant values are indicated (bold) in the table ( $\mathrm{p}<0.01^{* *}$ and $\left.\mathrm{p}<0.001^{* * *}\right)$.

\begin{tabular}{|c|c|c|c|c|c|c|c|}
\hline & \multicolumn{7}{|c|}{ aBMD } \\
\hline Formation markers & Arms & Legs & $\begin{array}{l}\text { Total } \\
\text { body }\end{array}$ & $\begin{array}{l}\text { Partial } \\
\text { body }\end{array}$ & $\begin{array}{c}\text { Total } \\
\text { hip }\end{array}$ & $\begin{array}{c}\text { Femoral } \\
\text { neck }\end{array}$ & $\begin{array}{c}\text { Lumbar } \\
\text { spine }\end{array}$ \\
\hline S-Bone ALP & 0.01 & -0.02 & 0.03 & 0.02 & -0.02 & -0.01 & 0.07 \\
\hline S-Total OC (N-Mid®) & -0.04 & $-0.22 * * *$ & -0.08 & -0.11 & 0.03 & -0.04 & -0.06 \\
\hline S-OC [1-49] & -0.05 & $-0.20 * * *$ & -0.09 & $-0.12 * *$ & -0.05 & -0.08 & -0.08 \\
\hline S-Total OC & -0.05 & $-0.21 * * *$ & -0.10 & $-0.12 * *$ & -0.04 & -0.08 & -0.07 \\
\hline S-cOC & -0.04 & $-0.22 * * *$ & -0.09 & $-0.12 * *$ & -0.06 & -0.08 & -0.05 \\
\hline \multicolumn{8}{|l|}{ Resorption markers } \\
\hline U-DPD/crea & -0.10 & $-0.19 * * *$ & $-0.15 * * *$ & $-0.15 * * *$ & -0.06 & -0.06 & 0.01 \\
\hline S-TRACP5b & -0.03 & $-0.19 * * *$ & -0.04 & -0.08 & -0.03 & -0.04 & -0.02 \\
\hline S-CTX-1 & -0.09 & $-0.21 * * *$ & -0.09 & $-0.12 * *$ & 0.02 & 0.05 & -0.02 \\
\hline \multicolumn{8}{|l|}{ Urinary osteocalcin } \\
\hline U-TotalOC/crea & -0.01 & -0.10 & 0.05 & 0.01 & 0.06 & 0.03 & 0.07 \\
\hline U-LongOC/crea & -0.06 & $-0.16 * * *$ & -0.06 & -0.09 & -0.08 & -0.08 & -0.07 \\
\hline U-MidOC/crea & -0.10 & $-0.16 * * *$ & -0.10 & $-0.12 * *$ & -0.06 & -0.07 & -0.04 \\
\hline
\end{tabular}


Table 3. Standardized regression coefficients $\left(\right.$ Beta $\left._{\text {std }}\right)$ between baseline levels of 11 bone markers and rate of bone loss, adjusted for baseline areal bone mineral density (aBMD) at the different regions of interest.

Significant values are indicated (bold) in the table ( $\mathrm{p}<0.01^{* *}$ and $\left.\mathrm{p}<0.001^{* * *}\right)$.

\begin{tabular}{|c|c|c|c|c|c|c|c|}
\hline & \multicolumn{7}{|c|}{ aBMD } \\
\hline Formation markers & Arms & Legs & Total body & Partial body & Total Hip & Femoral Neck & Lumbar Spine \\
\hline S-Bone ALP & -0.02 & 0.01 & 0.02 & 0.03 & -0.04 & -0.04 & 0.08 \\
\hline S-Total OC (N-Mid®) & -0.12 & $-0.20 * * *$ & -0.10 & -0.11 & $-0.11 * *$ & -0.10 & -0.06 \\
\hline S-OC [1-49] & $-0.13 * *$ & $-0.17 * * *$ & -0.11 & $-0.12 * *$ & $-0.13 * *$ & $-0.14 * * *$ & -0.08 \\
\hline S-Total OC & $-0.13 * *$ & $-0.18 * * *$ & $-0.12 * *$ & $-0.12 * *$ & $-0.12 * *$ & $-0.14 * * *$ & -0.07 \\
\hline S-cOC & $-0.12 * *$ & $-0.19 * * *$ & -0.11 & $-0.12 * *$ & $-0.15 * * *$ & $-0.15 * * *$ & -0.05 \\
\hline \multicolumn{8}{|l|}{ Resorption markers } \\
\hline U-DPD/crea & $-0.14 * *$ & $-0.17 * * *$ & $-0.16 * * *$ & $-0.15 * * *$ & -0.09 & -0.10 & 0.01 \\
\hline S-TRACP5b & -0.11 & $-0.16 * * *$ & -0.06 & -0.08 & -0.11 & $-0.11 * *$ & -0.01 \\
\hline S-CTX-I & $-0.16 * * *$ & $-0.18 * * *$ & -0.11 & $0.12 * *$ & -0.05 & -0.01 & -0.02 \\
\hline \multicolumn{8}{|l|}{ Urinary Osteocalcin } \\
\hline U-TotalOC/crea & -0.07 & -0.06 & 0.04 & 0.02 & 0.01 & -0.02 & 0.08 \\
\hline U-LongOC/crea & $-0.13^{* *}$ & $-0.13 * *$ & -0.08 & -0.09 & $-0.16 * * *$ & $-0.15 * * *$ & -0.07 \\
\hline U-MidOC/crea & $-0.16 * * *$ & $-0.13 * *$ & $-0.12 * *$ & $0.12 * *$ & $-0.14 * *$ & $-0.14 * * *$ & -0.03 \\
\hline
\end{tabular}


Table 4. Mean rate of bone loss (\% per year). Women in the highest quartile of a bone turnover marker (figure on the right) are compared to all other women (figure on the left). Statistically significant differences (t-tests) are indicated (bold) in the table $\left(\mathrm{P}<0.01^{* *}\right.$ and $\left.\mathrm{p}<0.001 * * *\right)$.

\begin{tabular}{|c|c|c|c|c|c|c|c|}
\hline & \multicolumn{7}{|c|}{ aBMD } \\
\hline Formation markers & Arms & Legs & Total body & Partial body & Total Hip & Femoral Neck & Lumbar Spine \\
\hline S-Bone ALP & $-0.89 /-0.94$ & $-0.54 /-0.66$ & $-0.32 /-0.33$ & $-0.44 /-0.50$ & $-1.32 /-1.32$ & $-1.49 /-1.59$ & $+0.35 /+0.46$ \\
\hline S-Total OC (N-Mid $\left.{ }^{\circledR}\right)$ & $-0.86 /-1.01$ & $-0.50 /-0.79 * * *$ & $-0.30 /-0.38$ & $-0.42 /-0.55$ & $-1.25 /-1.52$ & $-1.40 /-1.83$ & $+0.43 /+0.22$ \\
\hline S-OC [1-49] & $-0.84 /-1.02$ & $-0.50 /-0.79 * * *$ & $-0.29 /-0.42$ & $-0.40 /-0.59 * *$ & $-1.27 /-1.45$ & $-1.43 /-1.81$ & $+0.46 /+0.11$ \\
\hline S-Total OC & $-0.81 /-1.10 * *$ & $-0.50 /-0.80 * * *$ & $-0.28 /-0.44 * *$ & $-0.39 /-0.62 * *$ & $-1.25 /-1.52$ & $-1.41 /-1.87$ & $+0.45 /+0.14$ \\
\hline S-cOC & $-0.80 /-1.13 * *$ & $-0.49 /-0.81 * * *$ & $-0.28 /-0.45^{* *}$ & $-0.38 /-0.63^{* * *}$ & $-1.29 /-1.42$ & $-1.48 /-1.66$ & $+0.44 /+0.16$ \\
\hline \multicolumn{8}{|l|}{ Resorption markers } \\
\hline U-DPD/crea & $-0.81 /-1.10 * *$ & $-0.50 /-0.79 * * *$ & $-0.26 /-0.51 * * *$ & $-0.38 /-0.65 * * *$ & $-1.25 /-1.51$ & $-1.44 /-1.71$ & $+0.45 /+0.13$ \\
\hline S-TRACP5b & $-0.81 /-0.91$ & $-0.53 /-0.71$ & $-0.30 /-0.32$ & $-0.44 /-0.46$ & $-1.29 /-1.40$ & $-1.47 /-1.66$ & $+0.40 /+0.29$ \\
\hline S-CTX-I & $-0.84 /-1.08$ & $-0.50 /-0.78 * * *$ & $-0.30 /-0.40$ & $-0.41 /-0.58$ & $-1.32 /-1.32$ & $-1.50 /-1.53$ & $+0.40 /+0.29$ \\
\hline \multicolumn{8}{|l|}{ Urinary osteocalcin } \\
\hline U-TotalOC/crea & $-0.91 /-0.81$ & $\mid-0.55 /-0.63$ & $\mid-0.35 /-0.23$ & $\mid-0.47 /-0.39$ & $-1.35 /-1.20$ & $-1.55 /-1.44$ & $+0.30 /+0.59$ \\
\hline U-LongOC/crea & $-0.91 /-0.81$ & $\mid-0.53 /-0.69$ & $-0.32 /-0.31$ & \begin{tabular}{|l|}
$-0.44 /-0.47$ \\
\end{tabular} & $-1.25 /-1.52$ & $-1.43 /-1.78$ & $+0.46 /+0.13$ \\
\hline U-MidOC/crea & $-0.86 /-0.96$ & $-0.52 /-0.73 * *$ & $-0.30 /-0.39$ & $-0.41 /-0.55$ & $-1.25 /-1.51$ & $-1.41 /-1.84$ & $+0.42 /+0.24$ \\
\hline
\end{tabular}

